ISSN 0103-5150

Fisioter. Mov., Curitiba, v. 24, n. 4, p. 655-664, out./dez. 2011 Licenciado sob uma Licença Creative Commons

\title{
Conhecimentos dos usuários da Estratégia Saúde da Família sobre a fisioterapia
}

\author{
Knowledge about Physiotherapy of users \\ of Family Health Strategy
}

\section{Sarah Tarcisia Rebelo Ferreira de Carvalho ${ }^{[a]}$, Maria do Carmo Gullaci Guimarães Caccia-Bava ${ }^{[b]}$}

[a] Doutoranda em Ciências da Saúde pela Escola de Enfermagem de Ribeirão Preto (EERP/USP), Ribeirão Preto, SP - Brasil, e-mail: sarahtrfc@hotmail.com

[b] Profa. Dra. do Departamento de Medicina Social da Faculdade de Medicina de Ribeirão Preto (FMRP/USP), Ribeirão Preto, SP - Brasil, e-mail: mcbava@fmrp.usp.br

\section{Resumo}

Introdução: Muitas experiências exitosas de incorporação do fisioterapeuta no Programa Saúde da Família já foram publicadas na literatura. No entanto, alguns autores destacam que o desconhecimento da população sobre a fisioterapia é um dos fatores que limitam a atuação desse profissional na atenção básica. Objetivo: Essa pesquisa objetivou investigar o conhecimento dos usuários do Programa Saúde da Família sobre a Fisioterapia. Materiais e método: Trata-se de um estudo transversal, descritivo e quantitativo. Aplicou-se um questionário a 275 usuários de uma Unidade Saúde da Família em Ribeirão Preto/SP. Resultados: Foi constatado que a maioria dos entrevistados já tinha ouvido falar sobre a fisioterapia e a considerava importante. Entretanto, o conhecimento dos entrevistados voltou-se aos aspectos de reabilitação e/ou tratamento, ao se referirem: ao conceito de fisioterapia; indicarem que as pessoas que precisam de Fisioterapia são os deficientes físicos, portadores de lesão e/ou algias músculo-esquelético; que o fisioterapeuta trabalha principalmente no hospital e que não realiza ações educativas. Conclusão: Esses dados demonstram um conhecimento restrito do campo de atuação da fisioterapia. Dessa forma, evidencia-se a necessidade de execução de estratégias que visem maior divulgação do campo de atuação da fisioterapia.

Palavras-chave: Programa Saúde da Família. Conhecimentos. Fisioterapia. População. 
Abstract

Introduction: Many experiences of successful incorporation of the physiotherapist in the Family Health Program have been published in the literature. However, some authors point out that unfamiliarity of the public about Physiotherapy is one of the factors that limit the role of these professionals in primary health care. objective: This study investigated the knowledge of users of the Family Health Program about Physiotherapy. Materials and methods: This is a cross-sectional, descriptive and quantitative study. A questionnaire was given to 275 users of a Family Health Unit in Ribeirão Preto/SP. Results: It was found that most interviewees had heard about physiotherapy and considered it is important. However, knowledge of the interviewees turned to aspects of rehabilitation and/or treatment, when referring: to the concept of Physiotherapy; indicate that people who need physiotherapy are disabled, suffering from injury and/or types of pain in musculoskeletal; that the physiotherapist works primarily in the hospital and did not realize educational activities. Conclusion: These data demonstrate a limited knowledge of the field of physiotherapy. This research shows a need for implementation of strategies aimed at further dissemination of the field of Physiotherapy.

Keywords: Family health program. Knowledge. Physiotherapy. Population.

\section{Introdução}

O Ministério da Saúde, com a finalidade de reorientar o modelo assistencial do Brasil, criou, em 1994, o Programa Saúde da Família (PSF), reafirmando os princípios básicos do SUS (1). A partir da edição da Portaria n. 648/06, que trata da Política Nacional de Atenção básica, passa a ser denominado Estratégia Saúde da Família (ESF), buscando salientar a opção técno-política de transcender à lógica dos programas verticalizados e fragmentados, em busca da realização do cuidado integral, humanizado e resolutivo (2).

A ESF estrutura-se por meio das Unidades (USF) e Equipes de Saúde da Família, estas com composição mínima constituída por: médico generalista, enfermeiro, auxiliar ou técnico de enfermagem e Agentes Comunitários de Saúde (ACS). Cada equipe responsabiliza-se pelo acompanhamento de cerca de três a quatro mil pessoas, a quem deve garantir os fluxos de referência e contrarreferência aos serviços especializados, de apoio diagnóstico e terapêutico, ambulatorial e hospitalar (2). Essa operacionalização deve adequar-se a realidade local, desde que esteja baseada nos princípios e diretrizes fundamentais do SUS (3).

Reconhecendo a importância de outros saberes e práticas para consolidar a integralidade do cuidado, a Portaria n. 154/GM, de 24 de janeiro de 2008, propõe a criação dos Núcleos de Apoio à Saúde da Família (NASF) que devem atuar em conjunto com as Equipes de Saúde da Família e com toda a rede de serviços de saúde, objetivando ampliar o escopo de ações da ESF e, consequentemente, a sua resolubilidade. Os NASF vinculam-se a, no mínimo, oito e, no máximo, 20 Equipes de Saúde da Família, exceto nos Municípios com menos de 100 mil habitantes dos Estados da Região Norte. 0 fisioterapeuta pode integrar ambas as modalidades propostas (4).

Diante desse contexto, o advento da ESF fez surgir novas discussões em relação à atuação de outros profissionais na atenção básica. E entre esses profissionais, destaca-se o fisioterapeuta (5).

A origem da Fisioterapia no Brasil, entretanto, não teve uma tradição ligada à atenção básica à saúde. Ao contrário, historicamente concentrou suas ações principalmente nos níveis de atenção secundário referente ao diagnóstico e tratamento das doenças; e terciário - responsável pela limitação dos danos e reabilitação (6-10).

Isso ocorre provavelmente porque a Fisioterapia não apresenta um objeto de estudo ou de intervenção nitidamente definido, gerando absoluta atenção à doença e esgotando as possibilidades da profissão na tentativa de recuperar, reabilitar ou, ao menos, minimizar os sofrimentos (6). A formação acadêmica do fisioterapeuta também constitui entrave para romper o paradigma reabilitador, visto voltar-se, principalmente, para o atendimento em centros de reabilitação e hospitais (6-9).

Na contramão da sua origem, diante de uma concepção mais abrangente de saúde, associada à produção social e à qualidade de vida, o âmbito da Fisioterapia vem expandindo-se. Assim, gradativamente, 
o fisioterapeuta se integra à atenção básica, ampliando seu espectro de atuação para a promoção da saúde e prevenção de doenças (8-11).

No entanto, são vários os obstáculos que ainda impedem essa ampliação do campo de atuação do fisioterapeuta. Dentre eles, destaca-se a procura do serviço de Fisioterapia pela população. Robalo e Silva enfatizam que a população, por causa de aspectos socioculturais, muitas vezes, só recorre à Fisioterapia por necessidades consideradas urgentes e imediatas, tais como sequelas de Acidente Vascular Cerebral (AVC) ou dor lombar aguda (12). Esse fato desvaloriza o esforço e o investimento em ações de promoção da saúde, cujo resultado só é reconhecido em longo prazo e dificulta a concretização do princípio de integralidade da atenção.

Nesse sentido, estudo desenvolvido por Brasil et al. demonstrou que o desconhecimento a respeito da atuação do fisioterapeuta é motivo de limitação do acesso da comunidade ao serviço de Fisioterapia na ESF (13). Diante disse, citam-se alguns autores que ressaltam a necessidade de conscientizar os profissionais da área de saúde e a sociedade sobre a relevância do fisioterapeuta como agente de saúde $(5,8)$.

A Fisioterapia, regulamentada pelo Decreto-lei n. 938/69, Lei n. 6.316/75, Resoluções do Coffito, Decreto n. 9.640/84 e Lei n. 8.856/94, é definida como: "uma ciência da Saúde que estuda, previne e trata os distúrbios cinéticos funcionais intercorrentes em órgãos e sistemas do corpo humano" (14). Essa definição exclui dúvidas, antes existentes, sobre a capacidade desse profissional em ser inserido na atenção básica.

No Brasil, pesquisas indicam que o fisioterapeuta inicia sua participação na ESF em algumas regiões, como: Sobral-CE $(5,13,15)$, João Pessoa-PB (7), Teresópolis-RJ (9) e Belo Horizonte-MG (16). Entretanto, constata-se um número ainda restrito de estudos em relação à atuação do fisioterapeuta nessa estratégia.

Nesse contexto, o presente estudo objetivou investigar os conhecimentos dos usuários de uma USF de Ribeirão Preto-SP sobre a Fisioterapia. A Unidade em estudo conta com a atuação de uma equipe mínima da saúde que desenvolve atendimento ambulatorial de atenção básica, de baixa densidade tecnológica, visando à assistência da demanda espontânea e programada da população. Foi considerada como um campo adequado de pesquisa por seguir as diretrizes assistenciais do Ministério da Saúde, constituir-se de pessoas de diferentes níveis socioeconômicos e ter sido cenário de estudos anteriores $(17,18)$.

\section{Materiais e métodos}

O estudo com delineamento transversal e analítico desenvolveu-se resguardando os preceitos éticos de pesquisas em Seres Humanos (19), sendo aprovado pelo Comitê de Ética em Pesquisa do Centro de Saúde Escola da Faculdade de Medicina de Ribeirão Preto (protocolo n. 279).

Essa pesquisa teve como população-alvo usuários da Estratégia Saúde da Família da cidade de Ribeirão Preto-SP.

O cálculo do tamanho da amostra foi baseado no relatório Relação de Famílias por Microáreas, emitido pelo Sistema de Informação da Atenção básica (SIAB). Considerou-se uma população finita de 954 indivíduos, com proporção de $50 \%$ de acertos nas perguntas do questionário e grau de confiança de 95\%. Assim, a amostra desse estudo constituiu-se de 275 pessoas.

Foi entrevistada uma pessoa de cada família, selecionada de forma aleatória e sistemática, com base nesse relatório da SIAB. Estabeleceu-se que o entrevistado tivesse, obrigatoriamente, idade superior a 18 anos; estivesse presente na residência no momento da coleta de dados e tivesse sido atendido, pelo menos, uma vez na USF estudada. Na presença, no domicílio, de mais de uma pessoa que se enquadre nesses critérios, foi solicitado que indicassem entre eles a pessoa que iria participar. Na hipótese de que a família sorteada não apresentasse nenhum indivíduo que preenchesse os critérios de inclusão, a família residente no domicílio mais próximo à esquerda, que cumprisse as exigências, foi automaticamente incluída no estudo.

Utilizou-se como técnica de coleta de dados um questionário elaborado pela pesquisadora, contemplando a caracterização sociofamiliar e demográfica e os conhecimentos sobre a Fisioterapia. Após superação do período de pré-teste, o questionário foi aplicado por cinco ACS da Unidade em estudo, devidamente treinados pela pesquisadora. As entrevistas foram realizadas durante os finais de semana por duas razões: não interferir na rotina de trabalho dos agentes e para gerar a possibilidade de encontrar no domicílio não só a população feminina e do lar, mas, também, a população trabalhadora e de ambos os sexos. 
Utilizou-se análise estatística descritiva em que os dados foram categorizados e tabulados e os resultados foram expressos em frequências relativas e absolutas. As questões abertas e aquelas em que os entrevistados podiam citar mais de uma alternativa como resposta necessitaram de uma forma peculiar de apresentação. Optou-se por desenhar uma tabela discriminando os conceitos-chave ou as categorias e, para cada uma dessas opções, foram respondidos sim ou não. Dessa forma, foi possível saber quantas pessoas citaram cada alternativa.

\section{Resultados}

Em relação à caracterização sociofamiliar e demográfica, constatou-se que a metade dos usuários concentrava-se na faixa etária de 18 a 39 anos, com idade mínima de 18 anos e máxima de 79 anos, média de aproximadamente 43 anos, e desvio-padrão de 16. A população entrevistada foi essencialmente feminina (82\%), e em sua maioria se declarou parda $(59,6 \%)$, de religião católica $(54,2 \%)$ ou evangélica $(38,2 \%)$. Moravam com companheiros (58\%), trabalhavam no próprio lar (41\%) ou como prestadores de serviços em geral (33\%), apresentavam, no máximo, o ginásio incompleto (78\%) e pertenciam às classes econômicas C e D (86\%).

No tocante aos conhecimentos sobre a Fisioterapia, constatou-se que 247 entrevistados (90\%) já ouviram falar nela (Tabela 1), e que isso se deu por meio dos profissionais de saúde (51\%), meios de comunicação (41\%) e parentes e amigos (28\%). Considerouse essa questão como um fator decisivo e excludente na continuidade da entrevista. Dessa forma, do próximo tópico em diante foram incluídas apenas as respostas dos 247 indivíduos que já ouviram falar em Fisioterapia.

Tabela 1 - Distribuição dos usuários de uma Unidade de Saúde da Família por já ter ouvido falar em Fisioterapia - Sumarezinho, Ribeirão Preto (SP), 2008

\begin{tabular}{lcc}
\hline Já ouviram falar em Fisioterapia? & $\mathbf{n}$ & $\%$ \\
\hline Sim & 247 & 90 \\
Não & 28 & 10 \\
\hline Total & $\mathbf{2 7 5}$ & $\mathbf{1 0 0}$ \\
\hline
\end{tabular}

Em relação à autoavaliação do conhecimento sobre Fisioterapia, 70\% afirmaram querer saber mais sobre esse tema; $13 \%$ não tinham conhecimento algum sobre o assunto; $9 \%$ consideravam saber o suficiente; $5 \%$ não souberam responder e $3 \%$ não achavam necessário saber sobre isso.

Sobre o que seria fisioterapia, a metade dos entrevistados respondeu ser uma "forma de reabilitação, recuperação e/ou tratamento". Prevaleceu à relação da Fisioterapia com os termos "exercício, movimento, relaxamento e/ou massagem", em 34\%. Os que não sabiam responder configuraram 18\%. Dos entrevistados, $13 \%$ relacionaram a Fisioterapia aos benefícios, de forma geral, que esta proporciona, envolvendo as seguintes respostas: melhora a saúde, melhora o corpo, melhora a vida, é bom para saúde, ajuda as pessoas. A relação da Fisioterapia com a prevenção foi feita por apenas $4 \%$. Estiveram presentes com menor frequência, sendo categorizadas como "outras" (2\%), que a fisioterapia: estuda o metabolismo do organismo; é um aparelho; é medicação; é um exame (Tabela 2).

Referindo-se ao grau de importância da Fisioterapia, observou-se que $90 \%$ dos entrevistados consideraram a Fisioterapia muito importante e 7\% não souberam responder. Salienta-se, ainda, que as junções dos índices sem importância e pouco importantes somaram apenas 3\% do total.

A maioria dos entrevistados considerou como principais recursos que o fisioterapeuta utiliza: o exercício e/ou massagem (45\%) e os aparelhos e agentes elétricos e/ou térmicos (44\%). Salienta-se que a categoria "outros" incluiu as seguintes respostas: esteira, aparelho de pressão e medicamentos (Tabela 3).

As pessoas que precisavam dos serviços de Fisioterapia que mais foram mencionados pertenciam à categoria "deficientes físicos, lesões e/ou algias músculo-esquelética", com $76 \%$ dos entrevistados. A dimensão "acamados, idosos e doenças geriátricas" obteve $31 \%$, e as pessoas sadias só foram referidas por $2 \%$. As demais categorias também obtiveram pouco destaque (Tabela 4).

0 lugar de trabalho mais mencionado do fisioterapeuta foi o hospital, com $59 \%$ dos entrevistados. Em seguida, encontra-se a clínica, com $42 \%$. 0 posto de saúde foi citado por $38 \%$ dos pesquisados e o domicílio, por $15 \%$.

0 tipo de atividade do fisioterapeuta mais evidenciada foi à realizada individualmente (51\%). No entanto, as atividades de grupo adquiriram 48\%. A ativi- 
Tabela 2 - Distribuição dos usuários de uma Unidade de Saúde da Família por conceito de Fisioterapia, Sumarezinho, Ribeirão Preto (SP), 2008

\begin{tabular}{llrr}
\hline O que é Fisioterapia? & & $\mathbf{n}$ & \% \\
\hline Forma de reabilitação e/ou & Sim & 124 & 50 \\
tratamento & Não & 123 & 50 \\
& Total & $\mathbf{2 4 7}$ & $\mathbf{1 0 0}$ \\
\hline Exercício, movimento, & Sim & 84 & 34 \\
relaxamento e/ou massagem & Não & 163 & 66 \\
& Total & $\mathbf{2 4 7}$ & $\mathbf{1 0 0}$ \\
\hline Prevenção & Sim & 10 & 04 \\
& Não & 237 & 96 \\
& Total & $\mathbf{2 4 7}$ & $\mathbf{1 0 0}$ \\
\hline Relacionada aos benefícios & Sim & 33 & 13 \\
da Fisioterapia em geral & Não & 214 & 87 \\
& Total & $\mathbf{4 7}$ & $\mathbf{1 0 0}$ \\
\hline Psicoterapia & Sim & 06 & 02 \\
& Não & 241 & 98 \\
& Total & $\mathbf{2 4 7}$ & $\mathbf{1 0 0}$ \\
\hline Outros & Sim & 06 & 02 \\
& Não & 241 & 98 \\
& Total & $\mathbf{2 4 7}$ & $\mathbf{1 0 0}$ \\
\hline Não sabe responder & Sim & 45 & 18 \\
& Não & 202 & 82 \\
& Total & $\mathbf{2 4 7}$ & $\mathbf{1 0 0}$ \\
\hline & & & \\
& & & \\
& & &
\end{tabular}

dade domiciliar foi citada por $36 \%$ dos entrevistados, e as ações de educação em saúde, por apenas 9\%.

\section{Discussão}

As pessoas entrevistadas concentraram-se na faixa etária de 18 a 39 anos, estando em conformidade com os dados encontrados nos relatórios do SIAB de consolidação das famílias cadastradas do ano de 2008. No tocante ao sexo, a população entrevistada foi essencialmente feminina, e de valor semelhante aos $80 \%$ encontrados na pesquisa de Ferri (17).

0 fato de a maioria dos entrevistados já ter ouvido falar em Fisioterapia é relevante, por tratar-se de uma profissão relativamente nova, embora não seja suficiente para garantir a qualidade dos conhecimentos construídos sobre a profissão.
Tabela 3 - Distribuição dos usuários de uma Unidade de Saúde da Família por recursos que o fisioterapeuta utiliza, Sumarezinho, Ribeirão Preto (SP), 2008

\begin{tabular}{llrr}
\hline Recursos que o fisioterapeuta utiliza & n & \% \\
\hline Aparelhos e agentes elétricos & Sim & 108 & 44 \\
e/ou térmicos & Não & 139 & 56 \\
& Total & 247 & $\mathbf{1 0 0}$ \\
\hline Exercícios e/ou massagem & Sim & 112 & 45 \\
& Não & 135 & 55 \\
& Total & $\mathbf{2 4 7}$ & $\mathbf{1 0 0}$ \\
\hline Pesos & Sim & 06 & 02 \\
& Não & 241 & 98 \\
& Total & $\mathbf{2 4 7}$ & $\mathbf{1 0 0}$ \\
\hline Bolas & Sim & 57 & 23 \\
& Não & 190 & 77 \\
& Total & 47 & 100 \\
\hline Auxílio/marcha & Sim & 05 & 02 \\
& Não & 242 & 98 \\
& Total & $\mathbf{2 4 7}$ & $\mathbf{1 0 0}$ \\
\hline Outros & Sim & 17 & 07 \\
& Não & 230 & 93 \\
& Total & $\mathbf{2 4 7}$ & $\mathbf{1 0 0}$ \\
\hline Não sabe responder & Sim & 76 & 31 \\
& Não & 171 & 69 \\
& Total & $\mathbf{2 4 7}$ & $\mathbf{1 0 0}$ \\
\hline & & &
\end{tabular}

Nessa divulgação, os profissionais de saúde tiveram um importante papel, o que aponta para um dado essencial, visto que os profissionais necessitam ter conhecimentos sobre Fisioterapia para que possam detectar, na população, situações que exijam a atuação do fisioterapeuta e, assim, orientar as pessoas para os serviços existentes. Sobre isso, a pesquisa de Aguiar constatou que um em cada três dos profissionais das Equipes de Saúde da Família da área Distrital de Sumarezinho apresentou um bom grau de conhecimento sobre a atuação do fisioterapeuta (18).

Nessa mesma pesquisa, Aguiar mencionou que a Unidade em estudo já contou com a atuação de um fisioterapeuta voluntário, com permanência de uma vez na semana, voltada para reabilitação, realizando, principalmente, atividades domiciliares e individuais (18). Mesmo com atuação descontínua, esse pode ser um aspecto importante para que os entrevistados já 
Tabela 4 - Distribuição dos usuários de uma Unidade de Saúde da Família por pessoas ou situações que precisam dos serviços de Fisioterapia, Sumarezinho, Ribeirão Preto (SP), 2008

\begin{tabular}{llrr}
\hline $\begin{array}{l}\text { Pessoas ou situações que precisam } \\
\text { dos serviços de Fisioterapia }\end{array}$ & & \multicolumn{1}{c}{$\mathbf{n}$} & \multicolumn{1}{c}{$\%$} \\
\hline Pessoas sadias & Sim & 04 & 02 \\
& Não & 243 & 98 \\
& Total & $\mathbf{2 4 7}$ & $\mathbf{1 0 0}$ \\
\hline Acamados, idosos e doenças & Sim & 77 & 31 \\
geriátricas & Não & 170 & 69 \\
& Total & $\mathbf{2 4 7}$ & $\mathbf{1 0 0}$ \\
\hline Deficientes físicos/ lesões e/ou & Sim & 187 & 76 \\
algias músculo-esquelético & Não & 60 & 24 \\
& Total & $\mathbf{2 4 7}$ & $\mathbf{1 0 0}$ \\
\hline Doenças neurológicas & Sim & 19 & 08 \\
& Não & 228 & 92 \\
& Total & $\mathbf{4 7}$ & $\mathbf{1 0 0}$ \\
\hline Doenças respiratórias e cardíacas & Sim & 24 & 10 \\
& Não & 223 & 90 \\
& Total & $\mathbf{2 4 7}$ & $\mathbf{1 0 0}$ \\
\hline Necessidades estéticas & Sim & 03 & 01 \\
& Não & 244 & 99 \\
& Total & $\mathbf{2 4 7}$ & $\mathbf{1 0 0}$ \\
\hline Outros & Sim & 13 & 05 \\
& Não & 234 & 95 \\
& Total & $\mathbf{2 4 7}$ & $\mathbf{1 0 0}$ \\
\hline Não sabe responder & Sim & 26 & 11 \\
& Não & 221 & 89 \\
& Total & $\mathbf{2 4 7}$ & $\mathbf{1 0 0}$ \\
\hline & & & \\
& & & \\
& & &
\end{tabular}

tenham ouvido falar em Fisioterapia e por meio de profissionais de saúde. No entanto, o tipo de atividade realizada também pode ter influenciado uma ideia restrita do campo de atuação do fisioterapeuta.

Os meios de comunicação representaram um aspecto importante na disseminação de informações sobre a Fisioterapia. Esse dado foi inesperado, ao considerar-se que ainda não há uma mobilização efetiva dos Conselhos e Órgãos responsáveis pela divulgação da Fisioterapia para a sociedade, havendo poucas campanhas publicitárias veiculadas nos meios de comunicação. No entanto, o número de cursos de Fisioterapia é crescente em todo o país. Em Ribeirão
Preto, há uma faculdade estadual e quatro particulares que oferecem esse curso e, portanto, divulgamno pelos meios de comunicação, por eventos em praças públicas, shoppings, etc., com ações sociais em que são realizados exames simples (pressão, diabetes, etc.), exercícios e orientações.

A maioria dos entrevistados referiu que gostaria de saber mais sobre a Fisioterapia, sugerindo que essa divulgação ainda é superficial e, muitas vezes, de instância comercial, como a relacionada aos eventos das faculdades. Ao mesmo tempo, ratifica-se o interesse da população sobre o assunto, já que apenas $3 \%$ dos entrevistados demonstraram não achar necessário saber sobre o tema. A soma dos entrevistados que dizeram não ter qualquer conhecimento sobre a Fisioterapia com os que não souberam responder essa questão equivale a $18 \%$, sendo inferior ao encontrado na pesquisa de Blascovi-Assis e Peixoto, que constataram que $35,2 \%$ dos pacientes de Fisioterapia não tinham conhecimento algum sobre esse assunto antes de iniciar o tratamento (20).

Na questão "O que é fisioterapia?", destacou-se como grande referência a reabilitação e/ou tratamento, retomando o estigma essencialmente reabilitador que acompanha a Fisioterapia desde sua origem. Na pesquisa de Blascovi-Assis e Peixoto, também foi constatado que 53,9\% dos usuários de um serviço de Fisioterapia a relacionavam à reabilitação, à recuperação, aos recursos para evitar cirurgias, aos exercícios ou à forma de tratamento (20). No entanto, a visão atual da Fisioterapia é bem mais ampla, não se restringindo ao tratamento e reabilitação. Para Barros, o fisioterapeuta hoje diverge do conceito original, conquistando um novo campo legal e científico por meio da competência e do amadurecimento dessa categoria. Assim, o fisioterapeuta ampliou seu campo de atuação e deixou de ser um "profissional da reabilitação" para tornar-se um "profissional da saúde", atuante na promoção, no desenvolvimento, na prevenção, no tratamento e na recuperação da saúde (11).

A relação estabelecida entre Fisioterapia e exercício e/ou massagens, no presente estudo, converge com o estudo de Viana et al. realizado no Centro de Saúde São Gabriel, em que 54\% dos pacientes atendidos em um serviço de Fisioterapia associaram o tratamento fisioterápico à utilização de exercícios, ginástica e movimento. No entanto, esses pesquisados se referiram à Fisioterapia como um trabalho de nível técnico (16). 
Esse vínculo da Fisioterapia com o exercício está bem fundamentado, já que se trata de uma ferramenta-chave utilizada na maioria das atividades fisioterápicas com o objetivo de aprimorar a função e reduzir incapacidades $(21,22)$.

A maioria dos entrevistados considerou exercício e/ou massagem, e aparelhos e agentes elétricos e/ou térmicos como principais recursos que o fisioterapeuta utiliza. 0 estudo de Blascovi-Assis e Peixoto, em Sorocaba, que se diferenciou por entrevistar indivíduos em atendimento fisioterápico, verificou que o recurso mais utilizado também foi o exercício, categorizado como cinesioterapia $(73,3 \%)$, seguido da eletroterapia (60,52\%) (20). Assim, o conhecimento dos entrevistados na presente pesquisa sobre os recursos da Fisioterapia são de fato condizentes com os recursos utilizados na prática do atendimento.

No tocante às pessoas ou às situações que precisam de Fisioterapia, a maioria dos entrevistados mencionou a categoria de "deficientes físicos, lesão e/ou algias músculo-esquelético". Essa resposta pode ser justificada, de forma generalizada, pelos estudos que indicam a superior demanda de encaminhamento fisioterápico dos pacientes de ortopedia. A pesquisa de Viana et al. indicou que $43 \%$ dos pacientes atendidos em um serviço de Fisioterapia apresentavam diagnóstico ortopédico (16). No estudo de Blascovi-Assis e Peixoto, esse número correspondeu a $94,7 \%$. Para esses autores, a ortopedia inclui: traumatismos, lesões músculo-ligamentares, alterações posturais, etc (20).

A segunda categoria mais mencionada das pessoas ou situações que precisam de Fisioterapia abrangia "acamados, idosos, ou doenças geriátricas". Podese supor que esse fato seja resultante de uma visão cultural do idoso como um indivíduo doente, embora haja farta literatura indicando que a velhice não pode ser considerada como sinônimo de doença, mesmo sabendo que o aumento da idade é diretamente proporcional ao aumento do risco de comprometimento funcional e a consequente perda de qualidade de vida (23-25).

Embora os lugares de trabalho do fisioterapeuta aqui citados - o hospital e a clínica - também reforcem a ideia da Fisioterapia reabilitadora e ações com essa finalidade, o Posto de Saúde foi citado por 38\% dos pesquisados. Essa resposta surpreende porque a atuação do fisioterapeuta na atenção básica está em fase de implantação. Atualmente, no município, não há a presença desse profissional atuando diretamente nessas unidades. É valido lembrar que na área pesquisada já houve atuação de um fisioterapeuta de forma voluntária e que os entrevistadores desse estudo foram os ACS, fatores que podem ter influenciado nessa resposta.

A atuação individual do fisioterapeuta foi a mais mencionada, mas com pouca diferença em relação às atividades de grupo, sendo que essa visão pode estar relacionada ao grande número de atividades grupais realizadas na USF de referência para esse estudo, em que se realizam grupos com gestantes; de atividade física; de reeducação alimentar, de saúde da mulher, de quarteirões, de convivência, todos com boa adesão pela população. Alguns autores indicam que o trabalho de grupo tem se mostrado uma boa possibilidade de atuação da Fisioterapia na atenção básica $(7,10,13)$. Pesquisa de Viana et al. constatou que $67 \%$ dos usuários dos serviços de Fisioterapia participavam de atividades de grupo (16).

A atividade domiciliar do fisioterapeuta também foi mencionada e considerada importante diante das restrições para o deslocamento imposto por algumas doenças ou situações. Para Ragasson et al., o fisioterapeuta apresenta aptidões e competências inerentes à sua formação profissional, e uma delas é realizar atendimentos domiciliares em pacientes portadores de enfermidades crônicas e/ou degenerativas, pacientes acamados ou impossibilitados (26). Ribeiro também avalia esse tipo de atendimento como imprescindível, principalmente na atenção básica (7).

As ações de educação em saúde foram mencionadas por $9 \%$ dos entrevistados, podendo-se supor que a maioria dos pesquisados a considerara aparte do âmbito da Fisioterapia. No entanto, sabe-se que a Fisioterapia apresenta missão essencial por meio da aplicação de meios terapêuticos físicos, na prevenção, eliminação ou melhora de estados patológicos do homem, na promoção e na educação em saúde (26).

Outra justificativa para esse baixo índice relativo às ações de educação em saúde é a pouca valorização dessas atividades por parte da população (16, 27). A pesquisa de Ferri, realizada com os usuários da mesma USF, constatou que $66,2 \%$ deles procuravam os serviços dessa unidade, buscando pela consulta médica, e $25 \%$, para acompanhamento, prevenção de agravos e promoção da saúde (17). Assim, embora a maioria dos entrevistados dessa pesquisa ainda possa apresentar uma concepção hegemônica de saúde segundo o modelo curativo e reabilitador, 
já convive nesse território uma significativa adesão a uma visão mais abrangente de saúde e às ações de promoção e prevenção. Marin et al. também destacaram um grande avanço por parte da equipe de saúde e da população da USF Santa Augusta em Marília-SP em relação à adesão de ações dessa natureza, demonstrando que estão começando a compreender o conceito de saúde como qualidade de vida (27).

Diante desse cenário, evidencia-se que a atuação do fisioterapeuta no nível primário deve ocorrer em uma equipe multiprofissional e com uso de uma abordagem interdisciplinar, objetivando a realização de uma assistência integral e contínua. Deve seguir a lógica da territorialização, adscrição da população e responsabilização, e ocorrer, preferencialmente, no âmbito coletivo com a participação popular. As ações devem ser articuladas com diversos setores da sociedade e dos governos, considerando como prioritários a reversão dos determinantes e os condicionantes sociais da saúde (28).

\section{Considerações finais}

Acreditamos que o desconhecimento da população a respeito da atuação do fisioterapeuta, como demonstrado nessa pesquisa, é um importante motivo de limitação do acesso da comunidade a esses serviços. Mas acrescentamos que esse fato é agravado pela percepção, em uma esfera mais ampla, da saúde segundo o modelo hospitalocêntrico, de atenção centrado basicamente na doença, que se opõem aos princípios da Atenção Básica.

Diante disso, sugerimos o emprego de estratégias que visem a conscientizar a população e os profissionais de saúde sobre a importância de ações de promoção e prevenção à saúde. E, também, o incentivo da educação continuada para os profissionais de saúde, com uma atenção especial aos ACSs, para que esses possam identificar as necessidades reais da população, visto se tratar do membro da equipe de interação mais próxima à comunidade.

Especificamente sobre a Fisioterapia, acreditamos que, não havendo um valor dessa natureza arraigado na cultura, não serão geradas demandas nesse sentido, ao contrário da assistência médica, de reconhecimento consolidado e estimulado inclusive pelas superespecialidades.

A valorização e o incentivo que os serviços de atenção primária, geralmente, oferecem à participação ati- va da população, entretanto, podem gerar a produção de novos valores. Perante esse fato, ressaltamos a necessidade de ações conjuntas por parte dos diversos atores sociais envolvidos: conselhos e órgãos representativos da profissão, instituições formadoras e profissionais fisioterapeutas, que visem à divulgação da fisioterapia à população, demais profissionais da área da saúde e gestores, e facilitem o acesso da população a esses serviços.

Em relação aos Conselhos Federais e Regionais, destacamos a importância de realizar macro e micropolíticas bem estruturadas, ordenadas com base na realidade existente. Essas políticas devem ser capazes de elaborar e concretizar estratégias que visem a divulgar a Fisioterapia e o seu amplo campo de atuação, especialmente as ações de promoção e de prevenção, para contestar o seu paradigma essencialmente reabilitador e aproximar a atuação do fisioterapeuta à comunidade.

No que diz respeito às universidades e faculdades, sugerimos: maiores incentivos à pesquisa científica, com ênfase nas disciplinas relacionadas à Saúde Pública; inclusão de ações de prevenção e promoção em todas as disciplinas de sua grade curricular; incentivo a atividades em equipe multidisciplinares, com interação entre os diversos cursos e profissionais; estabelecimento de estratégias de interação entre as instituições formadoras e o Sistema de Saúde, de forma a aumentar o acesso ao atendimento fisioterápico à população e formar os futuros fisioterapeutas em novos campos de práticas hoje bastante restritos aos hospitais de ensino. Destacamos, ainda, que essa responsabilidade se estende a cada profissional fisioterapeuta, que deve contribuir com essa divulgação prestando atendimento de qualidade e desenvolvendo pesquisas que demonstrem a importância da atuação fisioterápica.

Estratégias como essas podem fazer com que o cidadão esteja cada vez mais apto a reconhecer suas próprias necessidades e possa solicitar os serviços mais compatíveis a elas. Acreditamos que pessoas mais cientes dos benefícios da Fisioterapia na promoção da saúde e na prevenção de doenças e agravos recorreriam à assistência antes da instalação de um quadro patológico, evitando muitos sintomas indesejáveis, doenças e sequelas. Mas, para que isso ocorra, faz-se necessário lutarmos em prol da expansão e do fortalecimento da Fisioterapia.

Diante desse contexto, essa pesquisa buscou contribuir no sentido de abrir caminhos e despertar o 
interesse de novos estudos para discutir a atuação do fisioterapeuta na atenção básica, como possibilidade de promover melhorias na qualidade da assistência e garantir a saúde como direito social fundamental da pessoa humana.

\section{Referências}

1. Brasil. Ministério da Saúde. Secretaria de Assistência à saúde. Coordenação de Saúde da Comunidade. Saúde da Família: uma nova estratégia para a reorganização do modelo assistencial. Brasília: Ministério da Saúde; 1997.

2. Brasil. Ministério da Saúde. Portaria n. 648/GM, de 28 de março de 2006. Aprova a Política Nacional de Atenção básica, estabelecendo a revisão de diretrizes e normas para a organização da Atenção básica para o Programa Saúde da Família (PSF) e o Programa Agentes Comunitários de Saúde (PACS). Diário Oficial [da] República Federativa do Brasil, Poder Legislativo, Brasília, DF, 2006, 28 mar, Seção 1. [acesso 5 ago. 2010]. Disponível em: http://dtr2001.saude.gov.br/ sas/PORTARIAS/Port2006/GM/GM-648.htm.

3. Dominguez BNR. O programa de saúde da família: como fazer. São Paulo: Computação Gráfica/Editora Ltda; 1998.

4. Brasil. Ministério da Saúde. Portaria n. 154/GM, de 24 de janeiro de 2008. Cria os Núcleos de Apoio à Saúde da Família - NASF. Diário Oficial [da] República Federativa do Brasil, Poder Legislativo, Brasília, DF, 2008, Seção 1, p. 38-42. [acesso 5 ago. 2010]. Disponível em: http://dab.saude.gov.br/docs/legislacao/ portaria154_24_01_08.pdf.

5. Véras MMS, Pinto VPT, Oliveira EN, Quinderé PHD. O Fisioterapeuta na Estratégia Saúde da Família: primeiros passos na construção de um novo modelo de atenção. SANARE [periódico na internet] 2004. [acesso 18 nov. 2007];5(1):169-73. Disponível em: http://www. esf.org.br/downloads/sanare/Sanare_v5_n1.pdf.

6. Rebelatto JR, Botomé SP. Fisioterapia no Brasil: fundamentos para uma ação preventiva e perspectivas profissionais. São Paulo: Manole; 1999.

7. Ribeiro KSQ. A atuação da fisioterapia na atenção primária à saúde: reflexões a partir de uma experiência universitária. Fisioterar Bras. 2002;3(5):311-18.
8. Afonso JL. Fisioterapia na atenção primária à saúde. Ícone. 1994;2(2):47-75.

9. Souza PCS. A fisioterapia e a construção de novos saberes e práticas a partir de Programa de Saúde da Família (PSF). Revista Crefito-2 [periódico na internet]. 2006 [acesso 20 jul. 2008];19:10. Disponível em: http://www.crefito2.org.br/Revistas/Revista19.pdf.

10. Veiga AC, Neves CAS, Montagna P, Kanda SS, Valença SS. A atuação do fisioterapeuta na unidade básica de saúde. Fisioter Bras. 2004;5(3):246-49.

11. Barros FBM. Autonomia profissional do fisioterapeuta ao longo da história. FisioBrasil. 2003;59:20-31.

12. Robalo L, Silva MG. A promoção e a proteção da saúde em Fisioterapia. EssFisiOnline [periódico na internet]. 2005 [acesso 20 jul. 2008];1(3):19. Disponível em: http://www.ifisionline.ips.pt/Arquivos_EssFisio_ files/vol1n3.pdf.

13. Brasil ACO, Brandão JAM, Silva MON, Gondin-Filho VC. O papel do fisioterapeuta do programa saúde da família do município de Sobral-Ceará. RBPS [periódico na internet] 2005 [acesso 20 nov. 2007];18(1): 3-6. Disponível em: http://www.unifor.br/notitia/ file/427.pdf.

14. Conselho Regional de Fisioterapia e Terapia Ocupacional. Leis e atos normativos das profissões de fisioterapeuta e do terapeuta ocupacional. Porto Alegre: CREFITO; 2001.

15. Pereira FWA, Mangueira JO, Monteiro MPA, Véras MMS, Lima VCS, Barrocas TCP, et al. A inserção da fisioterapia na estratégia saúde da família em sobral CE. SANARE [periódico na internet] 2004 [acesso 23 jul. 2007];5(1):93-100. Disponível em: http://www. esf.org.br/downloads/sanare/Sanare_v5_n1.pdf.

16. Viana SO, Merényi A, Sampaio RF, Furtado SRC. Fisioterapia na atenção primária: uma experiência de integração entre ensino, serviço de saúde e assistência à comunidade. Rev Bras Fisioter. 2003;7(2): 159-66.

17. Ferri SMN. As tecnologias leves como geradoras de satisfação em usuários de uma Unidade de Saúde da Família - elemento analisador da qualidade do cuidado prestado? [dissertação]. Ribeirão Preto: Escola de Enfermagem de Ribeirão Preto, Universidade de São Paulo; 2006. 
18. Aguiar RG. Conhecimentos e atitude sobre a atuação do profissional fisioterapeuta entre os profissionais da Equipe mínima de Saúde da Família em Ribeirão Preto [dissertação]. Ribeirão Preto: Faculdade de Medicina de Ribeirão Preto, Universidade de São Paulo; 2005.

19. Brasil. Conselho Nacional de Saúde. Resolução n. 196, de 10 de outubro de 1996. Dispõe sobre diretrizes e normas regulamentadoras de pesquisas envolvendo seres humanos. Diário Oficial [da] República Federativa do Brasil, Poder Legislativo, Brasília, DF, 1996. [acesso 10 ago. 2010]. Disponível em: http://dtr2004. saude.gov.br/susdeaz/legislacao/arquivo/Resolucao_ 196_de_10_10_1996.pdf.

20. Blascovi-Assis SM, Peixoto BO. A visão dos pacientes no atendimento de fisioterapia: dados para traçar um novo perfil profissional. Fisioter Mov. 2002; 15(1):61-7.

21. Hall CM, Brody LT, Taranto G. Exercício terapêutico: na busca da função. Rio de Janeiro: Guanabara Koogan; 2001.

22. Kisner C, Colby LA. Exercícios terapêuticos: fundamentos e técnicas. São Paulo: Manole; 2005.

23. Cianciarullo TI, Gualda DMR, Silva GTR, Cunha ICKO. Saúde na família e na comunidade. São Paulo: Robe; 2002.
24. Goldfarb DC. Corpo, tempo e envelhecimento. São Paulo: Casa do Psicólogo; 1998.

25. Simões R. Corporeidade e terceira idade: a marginalização do corpo idoso. São Paulo: Unimep; 1998.

26. Ragasson CAP, Almeida DCS, Comparin K, Mischiati MF, Gomes JT. Atribuições do fisioterapeuta no programa de saúde da família: reflexões a partir da prática profissional. [acesso 20 ago. 2006]. Disponível em: http://www.crefito5.com.br/web/downs/psf_ ado_fisio.pdf.

27. Marin MJS, Oliveira LR, Ilias M, Higa EFR. As contribuições da comunidade para o trabalho da equipe de um PSF. Rev Latino-Am Enferm. 2007;15(6):1065-71.

28. Bispo JP Jr. Fisioterapia e saúde coletiva: desafios e novas responsabilidades profissionais. Rev C S Col [periódico na internet] 2007. [acesso 10 abr. 2010]. Disponível em: http://www.abrasco.org.br/cienciae saudecoletiva/artigos/artigo_int.php?id_artigo = 1169 .

Recebido: 31/10/2010

Received: 10/31/2010

Aprovado: 30/05/2011

Approved: 05/30/2011 